\title{
Project THANKS: Examining HIV/AIDS-Related Barriers and Facilitators to Care in African American Women: A Community Perspective
}

\author{
Ndidiamaka Amutah-Onukagha, PhD, MPH, CHES, Meena Mahadevan, PhD, ljeoma Opara, MPH, MSW ${ }^{3}$ \\ Monica Rodriguez, MPH, Megan Trusdell, $\mathrm{MPH}^{4}$, and Jessica Kelly, $\mathrm{MPH}^{4}$
}

\begin{abstract}
Project THANKS, (Turning HIV/AIDS into Knowledge for Sisters), is an evidence-based intervention that utilizes a community-based participatory and empowerment building approach for African American female substance abusers living with HIV and other chronic diseases. This qualitative study sought to gain insight from women living with HIV on how to improve Project THANKS. African American women living with substance abuse disorders, HIV, and other comorbidities were recruited from three community based health centers in New Jersey $(N=31)$. Ninety minute focus group sessions were implemented in each health center. The focus group sessions were designed to understand the perceived factors influencing the participants' ability to selfmanage their health conditions and challenges they are currently facing regarding their diagnoses. The barriers and suggestions presented by participants included addressing stigmatization, managing mental health symptoms, improving physician-patient trust, accessing health education, educating community members, and proper nutrition. In addition, an engaged and trusting relationship with their healthcare provider and having positive sources of support were cited as motivators to adhering to their HIV treatment regimen. Participants living with HIV/AIDS also expressed more concern with difficulty treating their comorbidities than participants with only HIV/AIDS. Receiving input from African American women living with HIV related comorbidities was essential in improving the intervention to include a behavioral and primary health approach. Future programmatic interventions of Project THANKS will include a targeted focus on addressing mental health needs in women by offering meditation services and mental health referrals. In addition, Project THANKS will incorporate activities to improve communication with physicians, families, and media outlets to empower women to take an active role in their primary and social support needs.
\end{abstract}

Keywords: HIV/AIDS, community based participatory research, facilitators, African American women, comorbidities

\section{Introduction}

$\mathbf{R}$ ACIAL DISPARITIES IN HIV/AIDS diagnoses continue to negatively impact people of color who are disproportionately affected by the ongoing epidemic. According to the Centers of Disease Control and Prevention (CDC), African Americans represent only $12 \%$ of the population but represent $46 \%$ of HIV diagnoses in $2015 .{ }^{1}$ One out of 32 African American women will be diagnosed with HIV during their lifetime, while $64 \%$ of women living with HIV are African American. ${ }^{1}$ In addition, HIV/AIDS is the leading cause of death for African American women ages 25-44, and the likelihood of comorbidities is higher in HIV-positive persons than in HIV-negative persons. ${ }^{2,3}$ Nearly $30-50 \%$ of persons living with HIV/AIDS have current or past histories of drug and alcohol disorder. ${ }^{4}$ Substance abuse and HIV related comorbidities can often create a barrier to achieving a better quality of life in persons living with HIV/AIDS.

There are many public health challenges involved in the development and implementation of intervention programs designed for African American women living with HIV/AIDS. Existing interventions are often not culturally competent and

\footnotetext{
${ }^{1}$ Department of Public Health and Community Medicine, Tufts University School of Medicine, Boston, Massachusetts.

${ }^{2}$ Department of Nutrition and Food Studies, Montclair State University, Montclair, New Jersey.

${ }^{3}$ Department of Family Science and Human Development, Montclair State University, Montclair, New Jersey.

${ }^{4}$ Department of Public Health, Montclair State University, Montclair, New Jersey.
} 
are implemented without input from targeted groups. In addition, the involvement of multiple community based sectors and the social structural determinants that place women of color at risk of contracting HIV/AIDS are not fully addressed in treatment and in programming. Further, African American women living with HIV/AIDS are not a homogenous group and have unique within-group differences that are often ignored in intervention work. It is important for researchers to think more critically in legitimizing unique differences as it relates to African American women. Within-group differences pertain to the contextual factors that may have placed African Americans living with HIV/AIDS at risk (e.g., homelessness, intimate partner violence, substance abuse, mental health, and socioeconomic status). Such differences impact health outcomes for African American women living with HIV/AIDS.

\section{HIVIAIDS and medical adherence}

Lack of quality healthcare and mistrust of healthcare providers can become a deterrent in managing chronic illnesses such as HIV/AIDS. Patient satisfaction with healthcare providers has been found to be a motivating factor in medication adherence for African Americans. ${ }^{5}$ The patient/provider relationship can be negatively impacted by perceived racism and discrimination, which in turn can affect the decisions that African American women make regarding their health. ${ }^{6}$ Stories about unethical medical experiments such as the Tuskegee syphilis study have been passed down through multiple generations of African American families in the United States, and may contribute to beliefs and mistrust of the healthcare system. Studies examining the relationship between trust and perceived quality of medical care have discovered that African American women, regardless of sociodemographics, are more likely to report lower trust for their primary care provider. ${ }^{7,8}$

In addition, stereotypes by physicians of poor African American women can also negatively influence the quality of health information that physicians provide. For example, physicians caring for African American women may not feel the need to request in-depth medical information or may underestimate their patient's symptoms. ${ }^{8,9}$ African Americans are more likely to seek medical information from sources such as family, faith-based groups, and friends due to their perceptions of mistrust in healthcare provider advice. ${ }^{10,11}$ Specifically among persons living with HIV, the association of race and trust of healthcare providers significantly predicts nonadherence to antiretroviral therapy (ART). ${ }^{12}$ The resulting consequence of mistrust in medical providers leaves African American women living with HIV/AIDS at greater risk of developing complications that result in decreased quality of life and increased diagnosis of related comorbid diseases.

Exploring underlying issues in medical and medication adherence among persons living with HIV/AIDS can be complex but crucial in reducing negative health outcomes. Project THANKS was developed to explore these issues and provide women with the tools that are needed to understand why they are not able to adhere to medication so they can overcome such barriers.

\section{HIVIAIDS and comorbidities}

Individuals living with HIV/AIDS are at an increased risk of comorbid chronic and infectious diseases due to a number of factors. ${ }^{2}$ The advancement of antiretroviral medications
(ART) can produce side effects that result in insulin resistance, glucose intolerance, and metabolic syndrome, which have been found to be associated with obesity-related chronic diseases. $^{13}$

The prevalence of comorbidities in individuals living with HIV/AIDS has also been found to be higher in women than men. In one study, the most commonly self-reported comorbidities were as follows: diabetes, pulmonary disease, hepatitis $\mathrm{C}$, high blood pressure, and arthritis. ${ }^{14}$ Comorbidities can affect the health of individuals who are HIV positive and further exacerbate barriers to improving quality of life. Treatment for comorbid diseases tends to be more of a concern for individuals who are HIV positive. HIV-positive persons may be more focused on controlling the progression of HIV in their bodies, solely by prescribed ART medication, instead of attempting to treat other comorbid chronic diseases that they are living with. ${ }^{15}$

In addition to chronic and infectious diseases that are commonly found to be present in African American women living with HIV/AIDS, substance abuse continues to negatively affect adherence to treatment and impact quality of life. ${ }^{16-18}$ African American women, in comparison to other racial groups, are more likely to experience poverty, violence, gender discrimination, racial discrimination, and live in disorganized neighborhoods. ${ }^{19,20}$ These stressors often have negative physical and psychological consequences that may lead to maladaptive coping strategies such as using illicit substances. ${ }^{21}$

Substance abuse can significantly affect one's ability to make positive decisions regarding their health. Findings from the 2005 and 2009 National Survey on Drug Use for individuals living with HIV found that $64.44 \%$ had used an illicit drug during their lifetime and $27.9 \%$ had engaged in excessive alcohol binge drinking in the last month. ${ }^{22}$ Alcohol and substance abuse among HIV-positive persons, particularly those with a longer history of substance abuse, can affect cognition, inhibiting medication adherence, as well as treatment for comorbidities. 5,15

In addition to substance use, HIV can affect cognition by impairing the brain and central nervous system. Those with cognitive impairment are twice as likely to not follow medication guidelines. ${ }^{5}$ African American women living with HIV, especially those that are of lower socioeconomic status, who abuse substances, often do so as a coping mechanism for feelings of discrimination and stigmatization. ${ }^{23,24}$ Project THANKS was developed to effectively address this area as it is important to provide women living with HIV/AIDS with the skills and education that are needed to ensure they are able to live healthy lives.

\section{HIV and mental health}

Literature has shown that there is a strong association between substance abuse and mental illness, including major depressive disorder. ${ }^{25,26}$ In ethnic minority women and HIVpositive women, racial discrimination, gender discrimination, and stigma were found to be positively associated with depression. ${ }^{27}$ Mental health has also been shown to predict adherence to medications and the ability to make positive decisions regarding health. ${ }^{15}$ Substance abuse can also contribute to perceived discrimination and stigma against mental health, which is a persistent issue that widens health disparities among African Americans. Many may feel uncomfortable to 
disclose information regarding mental health symptoms to their primary care provider. Not only does depression affect medication adherence but also it can have an effect on the healthcare provider relationship between HIV-positive persons. ${ }^{28}$ Individuals living with HIV often report that they felt disrespected by their healthcare providers, which was significantly associated with increased depressive symptoms. ${ }^{29}$

Perceptions of stigmatization have been found to be higher among African Americans with a more recent diagnosis of HIV. ${ }^{30}$ Stigma has been noted as a reason that African Americans living with HIV resist seeking out care. ${ }^{31}$ Stigmatization among African Americans pertaining to HIV/AIDS testing and diagnosis has continued to contribute to the ongoing HIV/AIDS epidemic. Stigma in mental health treatment is still prevalent among African Americans, which makes treating comorbidities of mental illness and HIV/AIDS difficult in African American women. $^{32}$ The association of stigma and mental health symptoms in persons living with HIV/AIDS is well established in the literature. However, mental health symptoms due to the diagnosis of HIV/AIDS are often not addressed, leaving African American women living with HIV/AIDS with an unmet psychological need. Project THANKS provides education on how to alleviate the effects of the stigmatization of HIV/AIDS to improve emotional well-being in participants.

\section{Initial intervention}

Project THANKS (Turning HIV into New Knowledge for Sisters) was created and implemented for the purpose of supporting women living with HIV and comorbidities on how to adopt a healthier lifestyle through a series of workshops that focus on: (a) managing stress, (b) nutrition, (c) gaining social support, (d) encouraging health-seeking behaviors, and (e) promoting advocacy for better treatment programs and services. ${ }^{33}$ Project THANKS used the psychological and structural dimensions of empowerment to strengthen a participant's resolve to adopt healthy behaviors and to be an active participant in improving her own health. The program's culturally sensitive curriculum included components that have been adapted from CDC's compendium of evidence-based HIVprevention interventions for African American women, namely the Holistic Health Recovery Program (HHRP) ${ }^{34}$ and Sisters Informing Sisters about Topics on AIDS (SISTA). ${ }^{35}$

Like these interventions, Project THANKS is built on a foundation of fostering a sense of community among African American women living with HIV. Project THANKS differs from HHRP and SISTA as the curriculum for THANKS aims to provide women with the necessary knowledge and tools to manage the complications associated with having multiple chronic illnesses more effectively. Project THANKS was initially pilot tested with a group of 10 African American women that reported current drug and/or alcohol use and were HIV positive with at least one comorbidity. The results of Project THANKS have been previously documented. ${ }^{33}$

\section{Purpose of study}

In New Jersey, women account for about one-third of the adult/adolescent HIV/AIDS diagnoses in the state. In 2015, 86\% of new diagnoses among women occurred in minorities, and the rate of HIV/AIDS diagnosis among African American women was substantially higher than the rate among non-Hispanic white women. The primary modes of transmission among these wo- men are heterosexual contact $(55 \%)$ and injection drug use $(35 \%){ }^{36}$ It is estimated that the state will continue to spend hundreds of millions of dollars each year on medical costs to treat the medical complications associated with HIV and related illness costs that can be reduced through effective and comprehensive community-based services and education initiatives.

Essex County currently has the highest rate of HIV/AIDs cases in New Jersey. ${ }^{36}$ Newark, the most populated city, has the highest rate of HIV/AIDS cases in NJ and is located in Essex County. As of 2015, 70\% of people living with HIV/ AIDS resided in Essex County. Among Essex County female residents living in with HIV/AIDS, 82\% were African American/black women. ${ }^{36}$

This intervention aims to further examine barriers to treatment among African American women who are living with HIV and related comorbidities and to provide implications for improvements in Project THANKS and other interventions for HIV-positive women living with comorbidities. This study was conducted to provide women living with HIV/AIDS and comorbid disorders with the opportunity to improve the Project THANKS intervention. The importance of incorporating the suggestions and feedback from women living with the studied phenomena allows researchers to create effective programming that is relatable and adaptable to the targeted group.

\section{Methods}

\section{Setting}

Data were collected through a 90-min focus group in each of the three participating agencies: The North Jersey Community Research Initiative (NJCRI), St. Clare's, and Camden Area Health Education Center (AHEC), all located in New Jersey. NJCRI is one of the largest HIV/AIDS community based organizations in New Jersey. Its goal is to provide adequate care to clients living with HIV/AIDS in New Jersey, and to reduce social and health disparities among Newark, New Jersey, residents. NJCRI is also an advocate based organization and provides a list of comprehensive services to individuals living with HIV/AIDS, including substance abuse treatment, primary medical care, chronic disease management, health education, syringe exchange, food pantry, LGBTQ services, and homeless drop in shelter for adult and LGBTQ youth.

St. Clare's AIDS Resource Center, located in Newark, New Jersey, provides crucial support to individuals and families living with HIV/AIDS by providing clients with access to social services. St. Clare's provides comprehensive services such as case management, substance abuse prevention, mental health services, transitional housing, and emergency financial assistance. Camden AHEC is a part of the New Jersey AHEC that was established in 1978, in partnership with the former University of Medicine and Dentistry of New Jersey, the nation's largest health sciences university, which has now merged with Rutgers University. It is part of a national initiative to strengthen the health of communities through educational partnerships. Camden's AHEC serves primarily ethnic minorities, including black and Hispanic individuals.

\section{Procedure}

Purposive sampling was utilized as it was important for the study to ensure that participants all shared the same phenomena, which was living with HIV, using drugs, and/or being 
diagnosed with at least one other chronic illness. A phenomenological approach was used in this study to derive detailed narratives from participants describing their experiences.

Inclusion criteria included the following: (a) Englishspeaking African American women; (b) positive for HIV serostatus (documented by the facility staff through medical records or demonstration of prescriptions, medications, and medical appointments); (c) used an illicit drug and/or alcohol in the last 90 days (documented by self-report); (d) between ages 21 and 55 years; (e) have an additional chronic disease such as diabetes, hypertension, or heart disease(documented by the facility staff through medical records or demonstration of prescriptions, medications, and medical appointments); and (f) be willing to participate in a 90-min focus group.

During the initial contact, the facility's trained staff asked participants to complete a brief demographic questionnaire to record the participant's age, income, living situation, and medical history. Before administration of the questionnaires, informed consent was obtained. The facility staff was available to read the informed consent and the questionnaire items to those participants who may have had a difficult time filling them out on their own. As per IRB approval, all participants provided both written and verbal consent and stated that they were willing to participate in the study.

\section{Participants}

A total of 31 women participated in the study. All participants were women and identified as African American. Ages of the participants ranged from 21 to 67 years old, mean age of 50 years old. All women were diagnosed with HIV, substance abuse users, and had at least one chronic illness. Ten of the participants from all focus groups were high school graduates, and nine had less than a high school diploma. Six participants had technical or vocational school training, one participant had an associate's degree, four had some college experience, and one had a college degree. Nineteen participants were single, and five were in a long-term relationship. Four participants were widowed, and three were married.

\section{Researcher positionality}

The researchers engaged with many of the participants as advocates for their complex needs in the context of HIV/AIDS management and treatment through the implementation of the Project THANKS curriculum. In addition, we formed working relationships with the community based organizations from which we recruited the participants. As university faculty, we acknowledge that we come from privileged social locations. Although, as faculty of color, we come from diverse cultural and ethnic backgrounds, and can identify with intersectional perspectives of race and gender, we acknowledge that we are not experts on the daily issues and lived reality of the experiences of women living with HIV/AIDS and comorbid diseases in under resourced communities.

\section{Data gathering and analysis}

Focus groups were facilitated by the principal investigator and co-principal investigator of the Project THANKS intervention. The study intended to give control to participants in each group as much as possible to alleviate anxiety and create a safe space where they would allow themselves to feel comfortable to share their lived experiences. To establish trust and comfort within groups, both facilitators informally provided detailed information regarding our purpose and objectives for conducting the groups with the goal of ensuring participants that their responses would be used to improve the Project THANKS curriculum and provide implications for future intervention work with African American women living with HIV/AIDS and comorbidities. Participants were informed that they could withdraw at any time and that the session would be audio recorded.

The interview guide was developed as a result of pilot data that have been collected and reported previously (Mahadevan et al.). ${ }^{33}$ The topics that were covered include: (1) perceptions of healthy behaviors, (2) healthcare provider relationships, (3) facilitators and barriers to self-care and medication adherence, (4) sources of social support, and (5) suggestions for the development of an intervention that focuses on improving the overall health of HIV+ women with comorbidities.

Semistructured interviews were conducted in each of the three focus groups. Each focus group consisted of 9-12 women. Due to the complexity and sensitivity of the topic, questions were in depth and semistructured in format to allow for open discussion that pertains to the topic in addition to giving participants control of the session. All focus groups lasted $\sim 90 \mathrm{~min}$. Each focus group was conducted in private rooms in participating community organizations. During the interviews, facilitators probed the participants for improvements that they would like to see implemented in Project THANKS curriculum and the barriers that they feel have not been addressed in HIV/AIDS education curriculums. Each interviewer kept notes to refer back to for tacit and demographic information.

The primary and secondary author contributed to the coding. The codebook was constructed by the primary author, with review and validation from the secondary author. Grounded theory was utilized in the analysis. Saturation was reached, and the most salient themes that emerged were utilized. Thematic analysis was conducted by an independent graduate research assistant. The research assistant read the transcriptions inductively to see what themes emerged. Triangulation of notes and memos of analyses were exchanged among authors to ensure validity of the findings.

\section{Results}

\section{Challenges pertaining to the management of comorbidities}

While participants felt that taking care of their overall health is important, the majority of participants were more concerned with the treatment of their comorbidities. The participants felt that incorporating more strategies and highlighting the importance of managing common HIV related chronic illness would be beneficial in an intervention. Several participants mentioned struggling to manage various comorbidities, such as cholesterol, hypertension, poor mental health, kidney failure, diabetes, heart disease, and Alzheimer's disease, seizures, and arthritis.

\footnotetext{
"Because as long as you taking your medication as prescribed for your HIV, that's going to take care of that, okay, but if you have other illnesses going on, it's the HIV is not going kill you, it's the complications from the other illnesses..."
} 
“...high blood pressure, diabetes is not only from HIV. It's just, it's, we have to be more focused on it than someone that doesn't have the virus, cause that's for anybody, you know what I'm saying, that don't have it."

In addition to taking antiretroviral medication to treat HIV, the participants are prescribed various other medications to treat their comorbidities, which presented as a barrier to not only managing comorbidities but also properly adhering to multiple medications.

"The barriers is the amount of pills you take. Like when I was first diagnosed I was taking thirty pills. God bless me now, I take nine. But now I got arthritis so I am back to ten."

“There's some days that I just don't have no appetite and I can't take the medication without having something on my stomach because as she was saying it'll come back, and if doesn't come back it makes me feel sicker. So it's a struggle."

\section{Healthcare provider relationship}

Participants understood that adhering to their doctor's orders and medication regimen were a priority to achieve positive health outcomes. Some participants mentioned having a good relationship with their clinician and attributed their relationship to being able to control their health and adhere to their medication regimen.

"You have to be compliant with your healthcare and have a good relationship with your doctor" and several others agreed that they have good relationships with their doctors.

"Well, I happen to have a doctor and I love him. He does not rush, you know? You could be upset while you're sitting in the waiting room, but when I'm in his office, he doesn't rush. Matter of fact, I sit behind him and look at my charts because I want to know everything — what is that, why are you doing that, why is this high, whatever-and then, my questions I can pose to him, and then move on, but every doctor is not like that."

"She has bought into helping me improve my health, you know, it's not just about me coming in there and she reading over my labs and telling me what I'm high in, this that and the other, she helps me and she educates me on knowing about the disease of HIV. Not only that, there's a certain thing that I believe in that you have to have a spiritual connection with your doctor and you have to have a spiritual connection to the medication that you takin too."

Participants collectively agreed that they felt they needed more skills and strategies on how to effectively advocate for themselves to better understand their health. They felt this is an important component in Project THANKS and should be expanded more in the intervention. The participants agreed that it was important to learn assertive communication skills in discussing their health with their physician.

\footnotetext{
"You have to educate yourself, you have to advocate for yourself, if you feel like you're on too many medications, like, you need to understand-why am I on so many medications?"

“you have to participate in your own care, like, I don't like to accept what the doctors say...cause sometimes they can be wrong too... a lot of people think that whatever the doctor say, you got to do."
}

\section{Mental health}

While the participants described their overall health as good or fair, several indicated that their mental health is poor due to feelings of depression, anxiety, and stress. Some participants mentioned being diagnosed with bipolar disorder, which makes it difficult for them to adhere to their medications as prescribed. The participants felt that they struggled with their mental health, despite the treatment they received, including medication and/or psychological therapy. All of the participants that participated in the study were eligible to receive mental health services from the organizations they were recruited from, although many of the participants felt that the services were inadequate. Including mental health resources and management in future interventions was highly desired by participants.

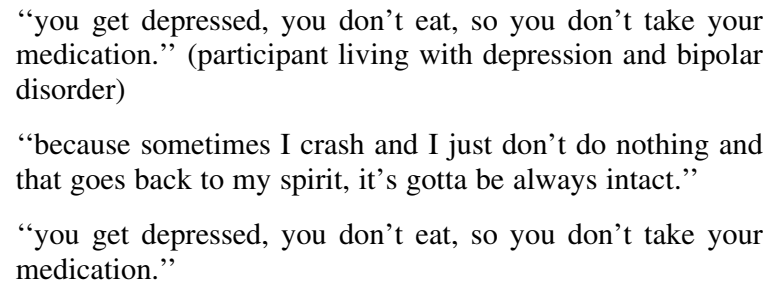

\section{Lack of social support}

The forms of social support varied for participants. Some indicated that they received support from family members, children, and their partner, while others felt they did not receive support from family. A challenge the women collectively faced was balancing their dual responsibilities in the family as mothers in addition to their HIV diagnosis. Many of the participants identified their children as a positive form of support in regard to managing their comorbidities.

"My daughter in college call me, 'Mom, you take your medicine?' If I sound under the weather, 'Okay, time to stay in the house, you know how you can get sick real quick.",

"You can live," you know, "It's in you but you live it," and that day on, "I took it from there and every week, that doctor used to call me just to see where I am."

Support groups in the community for women living with HIV allowed participants to connect with others that are also HIV positive according to participants. The participants felt that the people they met at support groups provided them with emotional support and a better understanding of living with HIV. Several participants described support groups as valuable for those who were recently diagnosed and unfamiliar with HIV, as a way to provide them with resources.

\footnotetext{
"Since I been coming here, you get to know, like, how other people living with the virus, you don't have to be alone, you know we do stuff together, and it's just a good thing."
}

While the participants indicated that they receive support from various sources, they felt stigmatized by the others due to their status. Participants would like to discuss stigmatization more in interventions as they feel HIV related stigma contributes greatly to their mental health. Participants mentioned hiding the fact that they were HIV positive due to stigma and how that adds more stress. One participant says:

\footnotetext{
"You sneaking, taking it. That's more stress. You find people that don't want nobody to know that they take pills. So now they go in the bathroom and someone might bust into the
} 
bathroom." Numerous participants state lack of education about HIV as a reason for stigma.

"... a lot of people cause of stigma are afraid to disclose, and to be honest, you need to get educate, and learn that you can't get HIV by hugging them, living next door to them, living in the same building...."

\section{Nutrition}

The participants expressed a need to incorporate lessons on how to eat healthy, culturally appropriate, and nutritious meals in interventions. Participants admitted that they were unaware of how to prepare healthy meals and felt they need not have access to nutritious foods in their communities. While some of the participants indicated that they try their best to eat healthy, HIV related comorbidities that they are living with sometimes prevent them from being able to. In addition to the importance of consuming a healthy diet, participants mentioned the need to eat to take their medication. The participants stated how they must consume food with certain medications, which may cause them to overeat or eat when they are not hungry.

\begin{abstract}
"Well as far as eating healthy, uh, with your, for your HIV, it depends on what other illnesses you have going on also, so it's like, you might be diabetic, so you have to watch your diet with that, your uh, your carbohydrates and stuff like that. You have to watch, you know, you know what you eat that way."

"I'm not going to say I take my medicine every single day because I don't. I might take my medicine five days out of the week. Some of it is because I don't eat. There's some days that I just don't have no appetite and I can't take the medication without something on my stomach..."
\end{abstract}

"Because I lose my appetite I do whatever it takes - I talk to a nutritionist - whatever my options is. If I can't eat this day, what can I take to get this medicine down because I'm going to get it down. No matter what."

\section{Access to health education}

Participants also expressed an interest in incorporating more educational workshops and programs that teach them how to effectively manage and understand their comorbidities. Topics of interest for educational workshops included: common HIV medication information and its side effects, healthy living strategies through a cultural lens, and having a better understanding of their laboratory results from their healthcare provider.

"I need to get better with learning about my numbers, remembering my numbers and knowing exactly where I am with things."

Another participant mentioned how religious organizations should provide information about HIV. The participants also expressed that the community/others should be educated about HIV to increase acceptance of them, described as:

\footnotetext{
"we just basically want to be treated like everybody else is being treated."
}

\section{Discussion}

The purpose of this study was to explore the lived experiences of African American women living with HIV, substance abuse disorder, and related comorbidities to evaluate and modify the existing intervention. Project THANKS was developed based on existing evidence-based behavioral interventions that address the needs of African American women living with HIV. The program includes additional components that help these women manage the challenges of HIV, as well as other chronic diseases such as diabetes, hypertension, heart disease, and cancer. ${ }^{33}$ Receiving feedback and input from African American women living with HIV, substance abuse disorder, and other comorbidities was essential in improving the Project THANKS intervention.

The findings of this study suggest that services for African American women living with HIV/AIDS should not only include more culturally sensitive education components but also seek to strengthen existing personal, social, and environmental support networks, which are needed to improve health outcomes among this population through a socioecological system lens. Utilizing an integrated health approach is crucial to improving the quality of life for African American women living with HIV and comorbidities due to the complexity of managing multiple illnesses.

The curriculum for Project THANKS will be modified in keeping with this approach. For example, program participants will be encouraged to engage in an open and honest dialogue to reflect on any personal factors (e.g., age, gender, income, education, substance use, medical diagnosis, and history of abuse) that may serve as barriers and decrease their motivation to adopt healthier dietary and lifestyle behaviors. They will be gently nudged to reflect on how these factors might make them feel helpless and disempowered and then discuss positive coping mechanisms to deal with them more effectively.

Social support is conceptualized as one of the most important antecedent stress resistance resources that contribute to positive adaptation outcomes. Many studies have examined the role of social support among women living with HIV and found that it is a significant predictor of medication adherence among HIV-positive women. ${ }^{37,38}$ While utilization of support groups has been shown to be an effective strategy in reducing feelings of perceived stigma and discrimination for women living with HIV, perceived stigma can present itself as a barrier to receiving and utilizing social support from family and loved ones. This can be attributed to feelings of shame and perceived disappointment, which then make it difficult to disclose one's status.

Project THANKS encourages its recipients to examine existing relationships with their partners, friends, or family members. The goal is to have them realize that an important part of pursuing a healthy lifestyle is to develop friendships and relationships with healthier boundaries. The modified curriculum will include additional role-playing and case scenario activities, which will impart specific communication strategies that allow them to openly express feelings of stigma and social isolation, reduce interpersonal conflict, and expand and foster healthier relationships within their social network.

Healthcare provider distrust is another complex issue that continues to be experienced by African Americans in particular. This can be due to perceived racial discrimination and physicians' lack of cultural competence and sensitivity. Participants indicated distrust in healthcare provider information, which may indirectly lead to lack of medical adherence to clinical recommendations. Interventions that address HIV-related stigma typically consist of educating individuals living with HIV/AIDS on how to address perceived stigma from healthcare providers. ${ }^{39}$ 
Project THANKS offers support to women in the program by educating participants on how to communicate effectively with healthcare providers regarding their health despite feelings of perceived discrimination. ${ }^{33}$ However, intervention experts caution that efforts to reduce stigma should be directed at everyone within a healthcare facility, including doctors, nurses, guards, cleaners, and administrative staff. This helps them to better understand the impact of HIV on their patients and families. ${ }^{40}$ Consistent with earlier studies, our study found that African American patients living with HIV/AIDS are more likely to adhere to medication and treatment if they feel that they have a good relationship with their physician. ${ }^{41-43}$ Interventions that combine individual-level information provision and/or skill building with organizationallevel activities, such as revising hospital policies, have been shown to present a more unified approach in the stigma reduction process. ${ }^{42-44}$ While Project THANKS is focused on providing information at the individual level, a key aspect of the program is to empower women to be proactive and advocate for improved public policies that will help HIV-positive women have access to better healthcare. Studies show that incorporating issue-specific community outreach knowledge and skills is critical for the intervention to succeed in preventing high-risk behaviors and reducing disease transmission. ${ }^{45}$

Using the American Public Health Association's Media Advocacy Manual as a guiding model, the women in Project THANKS learn to develop a step-by-step strategy to organize and educate each other, public officials, journalists, and the general public, on issues important to them, their families, and the communities in which they live. ${ }^{46}$ This includes creating a list of local media sources in their area, preparing a simple yet effective document showcasing their message clearly, writing a letter to the editor of a newspaper or magazine or TV channel explaining their message, arranging a meeting with the particular media source or using other public forums and meetings to present their issue, developing simple tips for following up with the source, and continuing to advocate for their cause.

Due to the participants having to manage HIV related comorbidities, medication management was another main theme that arose in all the focus groups. The participants expressed concern in learning how to manage HIV related comorbidities. They generally felt that while they were able to effectively manage HIV through proper medication adherence, managing complications associated with comorbidities was more challenging. Some of the other themes that emerged were related to difficulties navigating medical care, specifially adhering to multiple medications, seeing multiple doctors, and dealing with a lack of integrated healthcare. ${ }^{6,9}$

The modifications to Project THANKS will include a more comprehensive education on comorbidities and strategies for addressing these challenges. For example, some topics, such as the importance and urgency of making a lifestyle change for better overall health and the steps they should take to make these changes (gain more knowledge, abstain from drugs, manage stress, and adopt healthier dietary and exercise behaviors), will be incorporated because they are relevant to all women faced with a dual diagnosis of HIV and a chronic disease. The message that changing one's behavior (eating a balanced diet, being physically active, regularly self-monitoring blood glucose and blood pressure readings, watching out for heart attack symptoms, etc.) can influence the outcome (better control of diabetes, maintain normal blood pressure, and reduce heart disease risks) will be reinforced throughout the session.

The beneficial effects of setting goals in nutrition education and maintenance interventions are well established. The technique of setting a personalized goal has been shown to lead to measurable positive health outcomes, especially among individuals faced with challenging and difficult life circumstances. ${ }^{47}$ Project THANKS targets impoverished African American women with HIV who may be especially vulnerable to poor health outcomes associated with overweight/obesity and related chronic conditions due to a multitude of contextual factors, including limited resources, healthcare disparities due to racial discrimination and oppression, developmental histories marked by poor nutrition and disease, and psychosocial influences such as lack of self-efficacy, powerlessness, stigma, and feelings of social isolation. ${ }^{48,49}$

Thus, the program will aim to encourage each woman to set realistic goals for herself and outline specific tasks and activities that will help her accomplish these goals. Through a variety of hands-on games and activities, they will learn to identify a personal goal toward living a healthy lifestyle and then list, prioritize, schedule, and take the necessary action steps to achieve this goal. At the end of the session, the facilitator will encourage group members to continue to review and schedule a time for the next set of action steps that they would need to complete to achieve their goal. Minigroup sessions will also be convened throughout the program, in which the women will collectively come up with strategies to help them attain their goals. These group sessions will help them understand the importance of sharing their struggles in a group setting, develop social bonds with others, and hold one another accountable for change.

Another theme that emerged across all of the focus groups involved access to nutritional food options for women living with HIV/AIDS. Participants discussed the importance of receiving information about healthier eating options from their healthcare provider. Since the participants in the study were all living with HIV-related comorbidities, such as heart disease and diabetes, they expressed that food has a major role in managing their health effectively. Several women indicated that they were aware of which types of foods were bad for them (e.g., fried foods), however, lacked the ability to incorporate healthier alternatives into their diet.

In addition to HIV medication, all the women were taking other medications with varying side effects that either suppressed or increased appetite and induced nausea. Project THANKS addresses portion control using food models and models of silverware in different sizes. ${ }^{33}$ Nevertheless, incorporating healthier alternatives for women who suffer from nausea and low appetite would be included in the modification of the study as this is a primary concern to women living with HIV.

Upon being asked what the participants would like to see in an intervention program, most participants expressed an interest in more educational workshops and programs that would benefit their health. Topics of interest for educational workshops were medication and nutrition information. Patients also wanted a better understanding of their laboratory results from their healthcare provider. Topics that elicited additional feedback included information about their social 
networks receiving adequate education regarding HIV/AIDS, such as community networks, religious organizations, family, and peers, as they felt it would be helpful for them and reduce stigma.

Project THANKS includes components that have been adapted from CDC's compendium of evidence-based HIVprevention interventions for African American women, namely the HHRP and SISTA. Like these interventions, the intervention empowers women to be in charge of their own health by learning how to manage stress, adopt healthy coping mechanisms, and build social relationships based on mutual trust and respect. ${ }^{50,51}$ The program differs from HHRP and SISTA in that, in addition to HIV and substance abuse, the curriculum aims to provide women the necessary knowledge and tools to set a personal goal, adhere to the nutritional guidelines for HIV and other chronic diseases, manage complications associated with having multiple chronic illnesses more effectively, and advocate for improved public policies, programs, and services that provide them with access to better healthcare.

Research also shows that stigma plays a significant role in whether or not an individual feels supported and comfortable in disclosing their disease status, especially in settings that are generally considered safe, such as professional and medical settings. ${ }^{52}$ In a qualitative study comparing two groups of women with HIV (HIV well-managed vs. HIV not well-managed), researchers found that being treated with respect and empathy by their healthcare team was reported as being a critical influence on adhering to ART and for an overall sense of well-being. ${ }^{53}$

Based on the participant voices from focus group discussions in this study, potential modifications that will be made to the curriculum include improving the physician-patient relationship by encouraging participants to process their feelings regarding the medical care that they receive and how they perceive the relationship that exists with their physician. In addition, strategies should be established for better communication to foster trust between participants and physicians. Discussions on how to incorporate culturally appropriate healthier alternatives will occur to ensure adherence to a healthy diet, which is critical in chronic disease management.

Furthermore, the data reflect the need to partner with mental health service providers. Although Project THANKS does not provide mental health services through its intervention, trained facilitators will infuse stress relieving activities such as meditation into the curriculum to alleviate mental health symptoms. Incorporating strategies that can identify symptoms of mental health disorders and refer participants to mental health clinicians and services is essential in helping to reduce stigma and treat depressive symptoms, which can then increase medication adherence in participants.

\section{Limitations}

The authors acknowledge that there are limitations in this study; nonetheless, the findings of this study can contribute to further exploratory studies and literature pertaining to this field. It was important to utilize a qualitative approach to effectively capture the shared lived experiences of participants and provide them with the opportunity to contribute to modifications to the Project THANKS curriculum.

The sample design consisted of a nonrandomized sample of participants, who were already receiving services from the community health centers and consented to be a part of the study. Self-selection bias may be present due to the willingness of participants to agree to the study. Generalizability is limited in the study. The participants in the study were all African American women living with HIV, comorbidities, and substance abuse, which was the intended population for Project THANKS as it was tailored to serve this particular group. The goal of this qualitative research study was not generalizability, but rather to allow the specific target population of women to provide their valuable input that can be used to modify the Project THANKS intervention and enhance the scientific literature of effective interventions for increasing medication adherence and managing comorbidities in HIV+ women of color.

The results of the study were disseminated to community members through a variety of methods. Namely, the transcripts of the focus groups were shared with the participants at the organizations that they were recruited from, in the form of an open forum and dialogue. The participants were able to provide feedback and clarity regarding the information that was shared in the focus groups. The researchers felt that this triangulation of the data further strengthened the results and provided clearer implications of the lived experiences of the women in Project THANKS.

The results of the focus groups conducted can be applicable to other communities that comprise women of color living with HIV/AIDS and related comorbidities. We do not want to assume that low-income women living with HIV are a homogeneous group as there are within-group differences that should be acknowledged and taken into consideration. Further research among HIV-positive women with comorbidities and a substance abuse disorder in other communities is needed to effectively modify interventions to suit their needs.

Project THANKS contributes to the existing research on providing a continuum of care to women living with HIV, as they are guided through the process of prioritizing their health and HIV medication adherence. As stated in Mahadevan et al., ${ }^{33}$ Project THANKS aims to "sustain and build upon the community partnerships created for this project through implementation of the intervention at harm reduction agencies across the state and nationally, by creating new models of service and by providing 'on-site' trainings and workshops collaboratively with the agency staff." By providing insight to agencies and healthcare professionals, directly from the people they serve, meaningful and sustainable changes can emerge.

\section{Author Disclosure Statement}

No competing financial interests exist.

\section{References}

1. Centers for Disease Control and Prevention. HIV among African Americans. U.S. Department of Health and Human Services. 2017. Available at: https://www.cdc.gov/nchhstp/ newsroom/docs/factsheets/cdc-hiv-aa-508.pdf (Last accessed April 10, 2017).

2. Anzinger JJ, Butterfield TR, Angelovich TA, et al. Monocytes as regulators of inflammation and HIV-related comorbidities during cART. J Immunol Res 2014;2014:1-11.

3. Kelso GA, Cohen MH, Weber KM, et al. Critical consciousness, racial and gender discrimination, and HIV disease markers in African American women with HIV. AIDS Behav 2014;18:1237-1246. 
4. Durvasula R, Miller TR. Substance abuse treatment in persons with HIV/AIDS: Challenges in managing triple diagnosis. Behav Med 2014;40:43-52.

5. Thames AD, Moizel J, Panos SE, et al. Differential predictors of medication adherence in HIV: Findings from a sample of African American and Caucasian HIV-positive drug-using adults. AIDS Patient Care STDS 2012;26:621630.

6. Gaston GB, Alleyne-Green B. The impact of African Americans' beliefs about HIV medical care on treatment adherence: A systematic review and recommendations for intervention. AIDS Behav 2013;17:31-40.

7. Halbert CH, Kessler L, Wileyto EP, et al. Breast cancer screening behaviors among African American women with a strong family history of breast cancer. Prev Med 2006;43: 385-388.

8. Warren JR, Kvasny L, Hecht ML, et al. Barriers, control and identity in health information seeking among African American women. J Health Dispar Res Pract 2010;3:68-90.

9. Gaston GB. African-Americans' perceptions of health care provider cultural competence that promote HIV medical self-care and antiretroviral medication adherence. AIDS Care 2013;25:1159-1165.

10. Bogart LM, Wagner GJ, Green JD, et al. Medical mistrust among social network members may contribute to antiretroviral treatment nonadherence in African Americans living with HIV. Soc Sci Med 2016;164:133-140.

11. Sankar A, Neufeld S, Berry R, et al. Cultural rationales guiding medication adherence among African Americans with HIV/AIDS. AIDS Patient Care STDS 2011;25:547555.

12. Kalichman SC, Eaton L, Kalichman MO, et al. Race-based medical mistrust, medication beliefs and HIV treatment adherence: Test of a mediation model in people living with HIV/AIDS. J Behav Med 2016;6:1056-1064.

13. Lugassy DM, Farmer BM, Nelson LS. Metabolic and hepatobiliary side effects of antiretroviral therapy (ART). Emerg Med Clin North Am 2010;28:409-419.

14. Weiss JJ, Osorio G, Ryan E, Marcus SM, Fishbein DA. Prevalence and patient awareness of medical comorbidities in an urban AIDS clinic. AIDS Patient Care STDS 2010;24: 39-48.

15. Monroe AK, Rowe TL, Moore RD, Chander G. Medication adherence in HIV-positive patients with diabetes or hypertension: A focus group study. BMC Health Serv Res 2013;13:488.

16. Robinson AC, Knowlton AR, Gielen AC, et al. Substance use, mental illness, and familial conflict non-negotiation among HIV-positive African-Americans: Latent class regression and a new syndemic framework. J Behav Med 2016;1:1-12.

17. Gonzalez A, Mimiaga M, Israel J, et al. Substance use predictors of poor medication adherence: The role of substance use coping among HIV-infected patients in opioid dependence treatment. AIDS Behav 2013;17:168-173.

18. Mellins CA, Havens JF, McCaskill EO, et al. Mental health, substance use and disclosure are significantly associated with the medical treatment adherence of HIVinfected mothers. Psychol Health Med 2002;7:451-460.

19. Giurgescu C, Zenk SN, Dancy BL, et al. Relationships among neighborhood environment, racial discrimination, psychological distress, and preterm birth in African American women. J Obstet Gynecol Neonatal Nurs 2012; 41:51-61.
20. Perry Brea LA, Harp Kathi LH, Oser Carrie B. Racial and gender discrimination in the stress process: Implications for African American women's health and well-being. Sociol Perspect 2013;1:25-48.

21. Hurd NM, Varner FA, Caldwell $\mathrm{CH}$, Zimmerman MA. Does perceived racial discrimination predict changes in psychological distress and substance use over time? An examination among Black emerging adults. Dev Psychol 2014;50:1910-1918.

22. Substance Abuse and Mental Health Services Administration. The NSDUH Report: HIV AIDS and Substance Abuse, National Survey on Drug Use and Health 2010. Available at: http://www.samhsa.gov/sites/default/files/hivaids-and-substance-use.pdf (Last accessed April 12, 2017).

23. Neblett RC, Hutton HE, Lau B, McCaul ME, Moore RD, Chander G. Alcohol consumption among HIV-infected women: Impact on time to antiviral therapy and survival. J Womens Health 2011;20:279-286.

24. Davis EM, Galvan FH. Alcohol use among HIV-positive Latinas and African American Women. Affilia 2012;27: 435-448.

25. Lev-Ran S, Imtiaz S, Rehm J, et al. Exploring the association between lifetime prevalence of mental illness and transition from substance use to substance use disorders: Results from the National Epidemiologic Survey of Alcohol and Related Conditions (NESARC). Am J Addict 2013; 22:93-98.

26. Berger-Greenstein JA, Cuevas CA, Brady SM, Trezza G, Richardson MA, Keane TM. Major depression in patients with HIV/AIDS and substance abuse. AIDS Patient Care STDS 2007;21:942-955.

27. Logie C, James L, Tharao W, Loufty M. Associations between HIV-related stigma, racial discrimination, gender discrimination, and depression among HIV-positive African, Caribbean, and Black women in Ontario, Canada. AIDS Patient Care STDS 2013;27:114-122.

28. Jonassaint CR, Haywood C, Korthius PT, et al. The impact of depressive symptoms on patient-provider communication in HIV care. AIDS Care 2013;25:1185-1192.

29. Whetten K, Reif S, Whetten R, Murphy-McMillan LK. Trauma, mental health, distrust, and stigma among HIVpositive persons: Implications for effective care. Psychosom Med 2008;70:531-538.

30. Galvan FH, Davis EM, Banks D, Bing EG. HIV Stigma and social support among African Americans. AIDS Patient Care STDS 2008;22:423-436.

31. Arnold EA, Rebchook GM, Kegeles SM. 'Triply cursed': Racism, homophobia and HIV-related stigma are barriers to regular HIV testing, treatment adherence and disclosure among young Black gay men. Cult Health Sex 2014;16:710-722.

32. Okeke EN, Wagner GJ. AIDS treatment and mental health: Evidence from Uganda. Soc Sci Med 2013;92:27-34.

33. Mahadevan M, Amutah N, Ramos L, et al. Project THANKS: A socio-ecological framework for an intervention involving HIV positive African American women with comorbidities. J Health Dispar Res Pract 2014;7:87-105.

34. Margolin A, Avants SK, Warburton LA, et al. A randomized clinical trial of a manual-guided risk reduction intervention for HIV-positive injection drug users. Health Psychol 2013;22:223-228.

35. DiClemente RJ, Wingood GM. A randomized controlled trial of an HIV sexual risk reduction intervention for young African American women. JAMA 1995;274:1271-1276. 
36. New Jersey Department of Health. HIV/AIDS cases by groups statewide. Trenton, New Jersey.

37. Vyavaharkar M, Moneyham L, Tavakoli A, et al. Social support, coping and medication adherence among HIV+ women with depression living in rural areas of the southeastern United States. AIDS Patient Care STDS 2007;21: 667-680.

38. Cox LE. Social support, medication compliance and HIV/ AIDS. Soc Work Health Care 2002;35:425-460.

39. Mahajan AP, Sayles JN, Patel VA, et al. Stigma in the HIV/ AIDS epidemic: A review of the literature and recommendations for the way forward. AIDS 2008;22:67-79.

40. Ball K, Lawson W, Alim T. Medical mistrust, conspiracy beliefs and HIV-related behavior among African Americans. J Psych Behav Sci 2013;1:1-7.

41. Stangl AL, Lloyd JK, Brady LM, Holland CE, Baral S. A systematic review of interventions to reduce HIV-related stigma and discrimination from 2002 to 2013: How far have we come? J Int AIDS Soc 2013;16:18-34.

42. Nyblade L, Hong KT, Anh NV, et al. Communities Confront HIV Stigma in Viet Nam: Participatory Interventions Reduce HIV-Related Stigma In Two Provinces. Washington, DC: International Center for Research on Women (ICRW); 2008, pp. 1-37.

43. Apinundecha C. A community participation intervention to reduce HIV/AIDS stigma, Nakhon Ratchasima province, northeast Thailand. AIDS Care 2007;19:1157-1165.

44. Nyblade L, Stangl A, Weiss E, Ashburn K. Combating HIV stigma in health care settings: What works? J Int AIDS Soc 2009;12:15-22.

45. Freimuth VS, Quinn SC. The contributions of health communication to eliminating health disparities. Am J Public Health 2004;12:2053-2055.

46. American Public Health Association. Media Advocacy Manual. Catalog of Environmental Prevention Strategies. 2003;50. Available at: http://www.apha.org/NR/rdonlyres/
A5A9C4ED-1C0C-4D0C- A56C-C33DEC7F5A49/0/Media Advocacy_Manual.pdf (Last accessed April 12, 2017).

47. Cullen KW, Baronowsky T, Smith SP. Using goal setting as a strategy for behavior change. J Am Diet Assoc 2001; 101:562-566.

48. Adimora A, Schoenbach V, Doherty I. HIV and African Americans in the Southern United States: Sexual networks and social context. Sex Transm Dis 2006;33:39-45.

49. Hargreaves MK, Schlundt DG, Buchowski MS. Contextual factors influencing the eating behaviors of African American women: A focus group investigation. Ethnic Health 2012;7:133-147.

50. Margolin A, Avants SK, Warburton LA, Hawkins KA, Shi J. A randomized clinical trial of a manual-guided risk reduction intervention for HIV-positive injection drug users. Health Psychol 2003;22:223-228.

51. DiClemente RJ, Wingood GM. A randomized controlled trial of an HIV sexual risk reduction intervention for young African-American women. J Am Med Assoc 1995;274:12711276.

52. Fletcher F, Ingram LA, Kerr J, Buchberg M, Bogdan-Lovis L, Philpott-Jones S. "She Told Them, Oh That Bitch Got AIDS": Experiences of multilevel HIV/AIDS-related stigma among African American women living with HIV/AIDS in the South. AIDS Patient Care STDS 2016;30:349-356.

53. Brody LR, Jack DC, Bruck-Segal DL, et al. Life lessons from women with HIV: Mutuality, self-awareness, and selfefficacy. AIDS Patient Care STDS 2016;30:261-273.

Address correspondence to: Ndidiamaka Amutah-Onukagha Department of Public Health and Community Medicine Tufts University School of Medicine Boston, MA 02111-1845

E-mail: ndidiamaka.amutah_onukagha@tufts.edu 